\title{
Minichromosome maintenance complex component 8 and 9 gene expression in the menstrual cycle and unexplained primary ovarian insufficiency
}

\author{
Yelena Dondik $^{1,2}$ (D) $\cdot$ Zhenmin Lei $^{1} \cdot$ Jeremy Gaskins ${ }^{1} \cdot$ Kelly Pagidas $^{1}$
}

Received: 2 July 2018 / Accepted: 20 September 2018 / Published online: 1 October 2018

(C) Springer Science+Business Media, LLC, part of Springer Nature 2018

\begin{abstract}
Purpose DNA repair genes Minichromosome maintenance complex component (MCM) 8 and 9 have been linked with gonadal development, primary ovarian insufficiency (POI), and age at menopause. Our objective was to characterize $M C M 8$ and 9 gene expression in the menstrual cycle, and to compare $M C M$ 8/9 expression in POI vs normo-ovulatory women.

Methods Normo-ovulatory controls $(n=11)$ and unexplained POI subjects $(n=6)$ were recruited. Controls provided three blood samples within one menstrual cycle: (1) early follicular phase, (2) ovulation, and (3) mid-luteal phase. Six of 11 controls only provided a follicular phase sample. Amenorrheic POI subjects provided a single, random blood sample. MCM8/9 expression in peripheral blood was assessed with qRTPCR. Analyses were performed using delta-Ct measurements; group differences were transformed to a fold change (FC) and confidence interval (CI). Differences across menstrual cycle phases were compared using random effects ANOVA. Two-sample $t$ tests were used to compare two groups.

Results MCM8 expression was significantly lower at ovulation and during the luteal phase, when compared to the follicular phase $[\mathrm{FC}=0.69$ in the luteal vs follicular phase $(p=0.012, \mathrm{CI}=0.53,0.90)$; and 0.65 in the ovulatory vs follicular phase $(p=$ $0.0057, \mathrm{CI}=0.50,0.85)$ ]. No change in $M C M 9$ expression was noted throughout the menstrual cycle. No significant difference was seen in $M C M 8 / 9$ expression when comparing POI to control subjects.

Conclusions Our study showed greater $M C M 8$ expression in the follicular phase of the menstrual cycle, compared to the ovulatory and luteal phases. No cyclic changes were seen with $M C M 9$. Significant differences in $M C M 8 / 9$ expression were not detected between POI and controls; however, we recommend further investigation with a larger sample population.
\end{abstract}

Keywords Minichromosome maintenance complex · MCM8 · MCM9 · Primary ovarian insufficiency

\section{Introduction}

Human ovarian aging is related to the depletion of a preset primordial follicular pool that undergoes programmed cell death from conception until menopause [1]. A variety of factors affect this rate of follicular atresia, impacting a woman's reproductive lifespan. Most recently, genome wide association studies (GWAS) have suggested that gene loci associated with DNA repair pathways can also influence ovarian aging $[2,3]$.

Yelena Dondik

yelena.dondik@gmail.com

1 University of Louisville School of Medicine, Louisville, KY 40202 , USA

2 Division of Reproductive Endocrinology and Infertility, NYU Winthrop Hospital, Mineola, NY 11270, USA
Two such genes, Minichromosome maintenance complex component (MCM) 8 and 9 , have repeatedly been linked with gonadal development and reproductive life events [4].

Located on chromosome 20 and chromosome 6 respectively, MCM8 and MCM9 are necessary for repair of doublestranded DNA breaks (DSB) that occur during homologous recombination in meiosis I. Mouse models show gonadal failure due to arrested follicular development in $\mathrm{Mcm} 8$ deficient mice, and due to germ cell depletion in $\mathrm{Mcm} 9$ deficient mice [5]. Immunofluorescence studies in adult humans have also confirmed MCM8 expression in oocytes of primordial, primary, and secondary follicles [6]. Multiple studies have linked MCM8 single nucleotide polymorphism (SNP) rs16991615 with increased antral follicle count and delayed menopause onset in Caucasian women [6].

Primary ovarian insufficiency (POI) is defined as hypergonadotropic hypogonadism in a woman under 40 years 
old [7]. The incidence is approximately one in 100 women under the age of 40 and one in 250 women under the age of 35 . Although specific etiologies (i.e., Turner's syndrome, Fragile $\mathrm{X}$ premutation carrier, autoimmune disease) have been identified, 75 to $90 \%$ of cases of spontaneous POI remain unexplained. Recently, MCM 8 and MCM9 gene variants were found in a large cohort of patients with primary ovarian insufficiency [8]. Several other homozygous mutations in $M C M 8$ and MCM9 have also been reported in familial and consanguineous cases of POI [9-14]. Presumably, the POI phenotype occurs when dysfunction in MCM8 or 9 leads to impaired DSB repair in meiosis I, resulting in germ cell depletion and a dysgenic ovary [15].

Despite recent interest in these genes in the setting of human female fertility, no current data exists to detail the gene expression of MCM8 and MCM9 in the normal menstrual cycle. When examining $M C M 8$ gene expression in the peripheral blood of Arabic siblings with a homozygous $M C M 8 \mathrm{mu}-$ tation (c1954-1 G>A) resulting in primary gonadal failure, Tenenbaum-Rakover et al. [9] found a significant threefold decrease in $M C M 8$ gene expression when compared to unrelated normal controls, and an intermediate decrease when compared to heterozygous carriers in the same consanguineous family. However, there is no information available as to whether $M C M$ gene expression in women with POI is affected outside the setting of a specific familial mutation. The primary outcome of our pilot study was therefore to describe the potential variation of MCM8 and MCM9 gene expression in the normal menstrual cycle. The secondary outcome was to compare MCM8 and MCM9 gene expression in normal subjects and in females with unexplained POI.

\section{Material and methods}

\section{Subjects}

English-speaking Caucasian and Black patients were recruited from the Obstetrics and Gynecology department of a single academic medical center and informed consent obtained. Normal subjects were women aged 18 to 40 , with regular menstrual cycles (Q25-35 days), and without history of female-factor infertility or primary ovarian insufficiency (POI). Unexplained POI subjects were defined as those with amenorrhea or oligo-menorrhea related to repeatedly elevated follicle-stimulating hormone (FSH) levels in the menopausal range. All POI patients had a thorough clinical work-up excluding Fragile X premutation, Turner syndrome, and 21hydroxylase antibodies as a possible cause of POI, and were therefore considered "unexplained" at time of diagnosis. Subjects were also excluded if they had experienced medically related or surgical menopause (i.e., as a result of bilateral oophorectomy or cancer therapy) or had a personal history of galactosemia.

Demographic information (including age, race, menstrual cycle characteristics, gravidity and parity, maternal and sibling age at menopause, age at POI diagnosis) was collected for each subject.

Blood sampling was performed at three time-points in the normal menstrual cycle (early follicular phase - cycle day 2 4), at time of ovulation, and mid-luteal phase). Ovulation was determined with the use of a home urine-based ovulation predictor kit (OPK). Subjects were instructed on the use of the OPK and blood draw would occur 24-36 h after positive OPK. Brand of OPK used was based on user preference as no single brand is recommended by our clinic for monitoring for the Luteinizing hormone (LH) surge. Normal subjects who were not able to provide multiple blood samples were drawn only in the follicular phase. Alternatively, all of the POI patients were noted to be amenorrheic and therefore only underwent a single blood draw. All whole blood samples were collected using DNA/RNA shield blood collection tubes (Zymo Research, CA, USA). Samples were then aliquoted and stored at $-80^{\circ} \mathrm{C}$ until further lab studies were performed.

\section{RNA extraction and quantification}

RNA was extracted from whole blood samples stored in DNA/ RNA shield using Quick-RNA Whole Blood kits according to manufacturer's instructions (Zymo Research, CA, USA). First-strand cDNA synthesis was then performed using the High Capacity cDNA Reverse Transcription kit following the protocol recommended by the manufacturer (Applied Biosystems, CA, USA). MCM 8 and MCM9 mRNA expression was assessed with quantitative real-time PCR using SYBR Select Master Mix (Applied Biosystems) and primers for human MCM8 and human MCM9. MCM8.1 and MCM9.1 primer sequences were obtained from PrimerBank (https:// pga.mgh.harvard.edu/primerbank/index.html). All additional primers were designed using the NCBI Primer-Blast web tool (https://www.ncbi.nlm.nih.gov/tools/primer-blast/) to target regions of previously published MCM8 and MCM9 mutations (Table 1). The Step One Plus Real-Time PCR system (Software v2.2.2, Applied Biosystems) was used. Reverse transcribed cDNA samples in dilutions of 1:2 were used and at least two separate assays were performed for each sample. The quantity of MCM8 and MCM9 mRNA were normalized to human RPL19 in the same assay.

\section{Statistical analysis}

Subject characteristics of POI females vs normal controls were compared using Chi-square test for categorical variables and one-way ANOVA for continuous variables. To compare the difference across different menstrual cycle phases, a random 
Table $1 \quad M C M 8$ and $M C M 9$ primers

\begin{tabular}{|c|c|c|c|}
\hline Primer & Forward $\left(5^{\prime}-3^{\prime}\right)$ & Reverse $\left(5^{\prime}-3^{\prime}\right)$ & Comments [8-14] \\
\hline MCM8.1 & $\begin{array}{l}\text { TTTACAGCGATAGCTCTCCT } \\
\text { TTG }\end{array}$ & AGGTGCATCTCTTAGTTCAGTTG & PrimerBank ID: $33469925 \mathrm{c} 3$ \\
\hline MCM8.2 & AAAACCTTGGCTTGCATGGG & CATAGTTGTACACCCTTGCATGAAT & targets missense mutation at c. 950 \\
\hline MCM8.3 & GCGGAAGAAGGTTCTCGAAAT & GGTCTTTAAGTGAGAACTCCATCA & targets frameshift mutation at c. $1469-1470$ \\
\hline MCM8.4 & ATTCCAATTCGGGGAGACCC & $\begin{array}{l}\text { CGATTCCACAAATACCTTGA } \\
\text { TCACC }\end{array}$ & $\begin{array}{l}\text { targets missesense mutation c. } 1802 \text { and splice } \\
\text { mutation at c. } 1954\end{array}$ \\
\hline MCM9.1 & TCCCCAGTGAAGTGCTTACAA & CCCGCTCAAACTCCAGAACC & PrimerBank ID: 312284068c2 \\
\hline MCM9.2 & ACTTGCCTCTCAGGCTTGTC & GTGAGGTCATCACCAGATTTGC & targets frameshift c. $672-673$ \\
\hline MCM9.3 & GCAAGCTGAACACAAGGACC & CTCTGATTTGCTTGGGTAACCTTT & targets stop codon@ c.1483 \\
\hline MCM9.4 & AATCTGCAGCCCACACTGTC & ATCAGGCGAGCATGAGCTTC & $\begin{array}{l}\text { targets } 2 \text { mutations, c1651 STOP and c1732 } \\
\text { ABNORMAL SPLICE SITE }\end{array}$ \\
\hline
\end{tabular}

effects ANOVA analysis was performed. Two-sample $t$ tests were used to perform comparisons between two groups. All analyses were performed using delta-Ct measurements, and group differences were transformed to a fold change and confidence interval for interpretation. All statistical analysis was performed using the R statistical software. This study was approved by the University of Louisville Institutional Review Board (IRB\# 16.0862).

\section{Results}

A total of six POI subjects and 11 control subjects were enrolled in the study (See Fig. 1). As the initial study protocol did not exclude normal subjects on hormonal contraception as long as they had documented fertility assessment and regular menstrual cycles within 1 year of the study, four of the 11 control subjects were using hormonal birth control (one on oral contraceptive pills, three with Progestin intrauterine device) at the time of their blood draw. All control subjects had a blood draw in the early follicular phase of the cycle. Five of the control subjects submitted three blood samples per menstrual cycle, to investigate with-in cycle changes in gene expression. None of these five patients used hormonal birth control. Patient characteristics of the two study groups are compared in Table 2, confirming no significant difference in age and race between POI and control subjects (regardless of hormone use) (Table 2). Of note, no study subject reported a family history of POI. All subjects reported

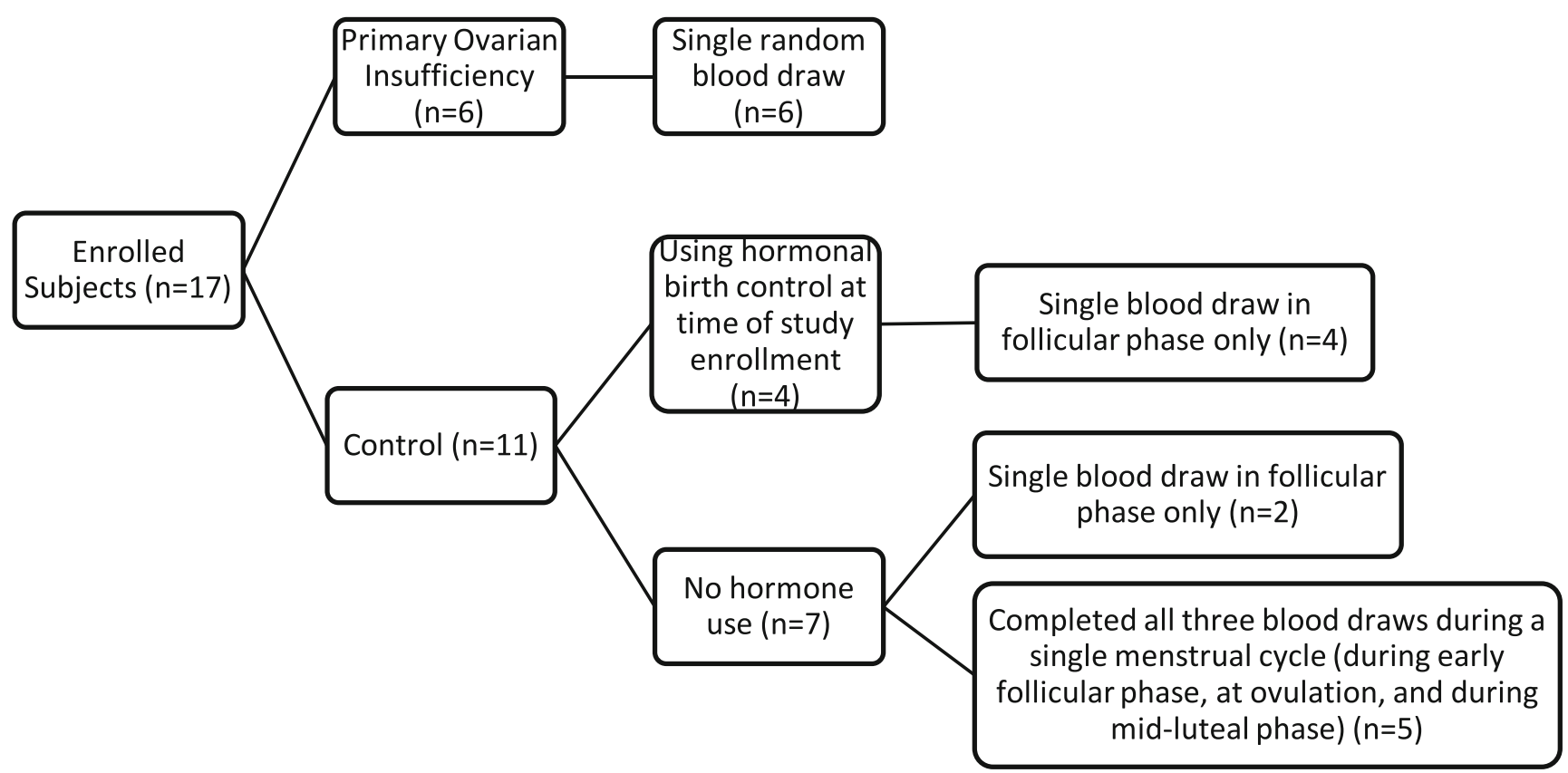

Fig. 1 Study enrollment 
Table 2 Characteristics of study participants

\begin{tabular}{lrclc}
\hline Characteristic & POI $(n=6)$ & All controls $(n=11)$ & $\begin{array}{l}\text { Controls without hormonal } \\
\text { contraception }(n=7)\end{array}$ & $P$ value \\
\hline $\begin{array}{l}\text { Age (years) (Mean, SD) } \\
\text { Race }(n, \%)\end{array}$ & $28.5(6.2)$ & $30.5(4.1)$ & $30(4.7)$ & $0.7296^{\mathrm{a}}$ \\
Caucasian & $4(66.7 \%)$ & $10(91 \%)$ & $6(86 \%)$ & $0.431^{\mathrm{b}}$ \\
Black & $2(33.3 \%)$ & $1(9 \%)$ & $1(14 \%)$ & \\
\hline
\end{tabular}

${ }^{a}$ One-way ANOVA comparing POI vs all controls vs controls without hormonal contraception

${ }^{\mathrm{b}}$ Chi-square maternal age at menopause as being greater than 50 years old, or menopause not yet achieved when maternal age was less than 50 .

As noted above, qRTPCR was performed using multiple primers for the genes of interest. However, cycle threshold $(\mathrm{Ct})$ values were consistently very high (between 33 and 37 ) for the MCM8.1, 8.2, and 8.4 primers, despite amplification of all samples and consistent $\mathrm{Ct}$ for each subject regardless of primer. Alternatively, MCM8.3 showed $\mathrm{Ct}$ values ranging from 32 to 34 . Due to this observed difference, we are including our data analysis for both the MCM8.1 and MCM8.3 PCR
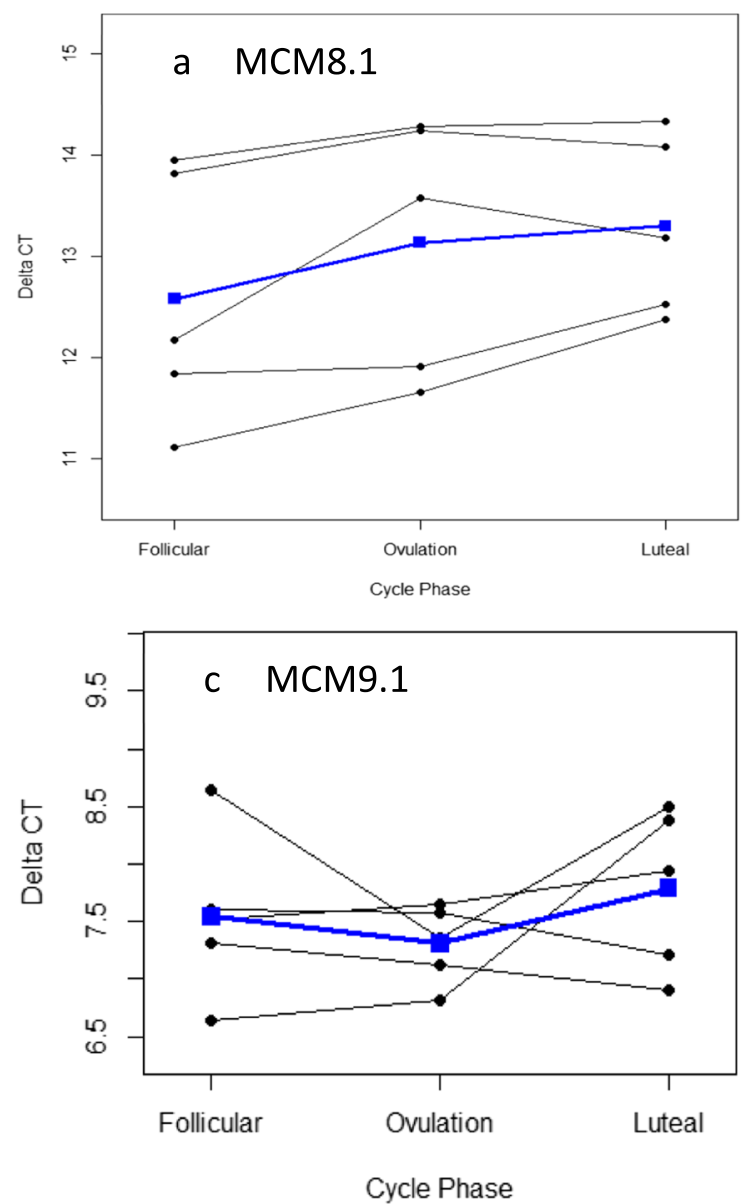

results. All MCM9 primers generated consistent amplification and $\mathrm{Ct}$ values across subject samples; therefore, data analysis was performed using the MCM9.1 primer data.

\section{Menstrual cycle analysis}

A significantly lower $M C M 8$ gene expression was noted in the non-follicular phases, when compared to the early follicular phase (Fig. 2a, b). Using primer MCM8.1, the fold change was 0.60 in the luteal vs follicular phase $(p=0.0089, \mathrm{CI}=0.43$, $0.85)$; and 0.68 in the ovulatory vs follicular phase $(p=$

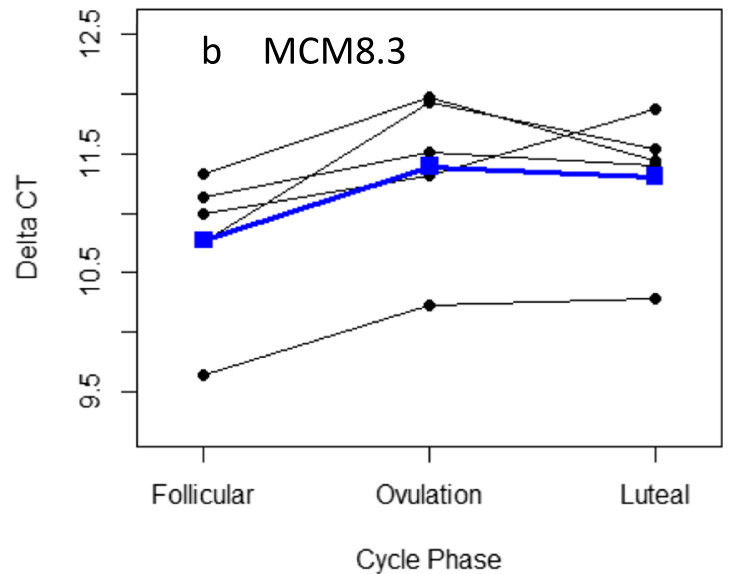

Fig. 2 Delta Ct for primers MCM 8.1 (a), MCM 8.3(b), and MCM9.1 (c) in the follicular, ovulatory, and luteal phases of the menstrual cycle. Blue line represents mean values 
$0.0292, \mathrm{CI}=0.49,0.95)$. Using primer $M C M 8.3$, the fold change was 0.69 in the luteal vs follicular phase $(p=0.012$, $\mathrm{CI}=0.53,0.90)$; and 0.65 in the ovulatory vs follicular phase $(p=0.0057, \mathrm{CI}=0.50,0.85)$.

No significant change in $M C M 9$ gene expression was noted throughout the menstrual cycle phases (Fig. 2c). The fold change was 0.84 in the luteal vs follicular phase $(p=0.512$, $\mathrm{CI}=0.48,1.49)$ and 1.18 in the ovulatory vs follicular phase $(p=0.523, \mathrm{CI}=0.67,2.07)$.

\section{Effects of hormonal contraception on gene expression}

When comparing follicular phase samples of control subjects on hormonal contraception $(n=4)$ to those without hormonal contraception $(n=7)$, no significant difference in gene expression was noted in MCM8. MCM8.1 showed fold change of $0.74(p=0.5585, \mathrm{CI}=0.24,2.30)$, and MCM8.3 showed fold change of 1.03 ( $p=0.9245, \mathrm{CI}=0.56,1.87)$. While the difference in MCM9 gene expression was also not statistically

\section{a $\quad$ Delta CT for MCM8.1}

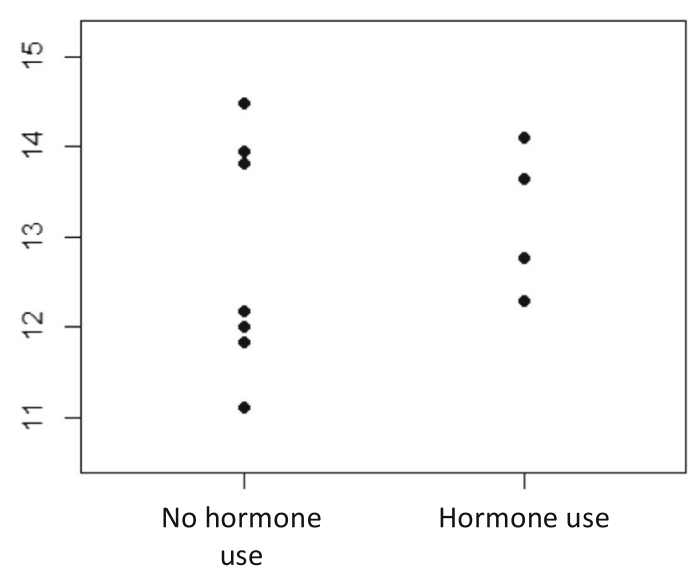

C Delta CT for MCM9.1

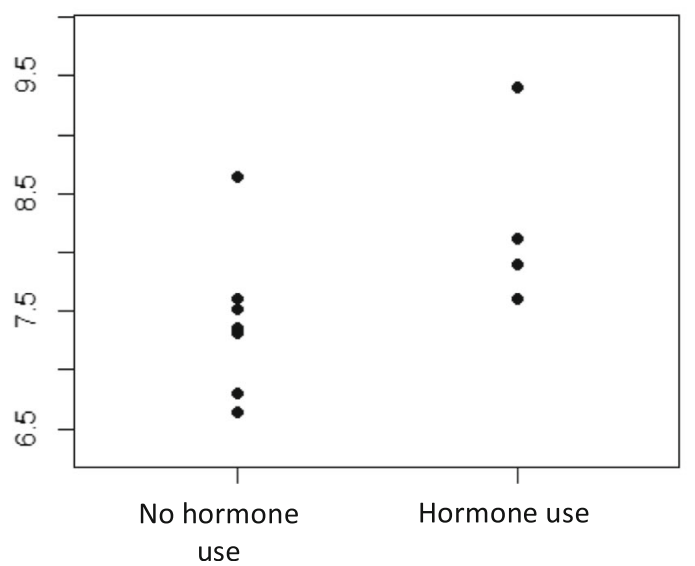

significant between the two groups, it is possible that a significant difference would have been seen with a larger sample size $[M C M 9$ fold change $=0.56(p=0.0865, \mathrm{CI}=0.28,1.10)]$. (Fig. 3).

\section{POI vs normal control subjects}

There was no statistically significant difference seen in MCM8 or MCM9 expression when comparing POI subjects $(n=6)$ to control subjects $(n=11)$ in the follicular phase (Fig. 4). MCM8.1 showed fold change of 1.01 ( $p=0.9876, \mathrm{CI}=0.47$, $2.14) ; M C M 8.3$ showed fold change of $1.11(p=0.5875, \mathrm{CI}=$ $0.74,1.65)$, and MCM9 showed fold change of 0.75 ( $p=$ $0.316, \mathrm{CI}=0.41,1.36$ ). When control subjects on hormonal birth control were removed from the analysis, the results remained consistent. MCM8.1 showed fold change of 0.9 ( $p=0.804, \mathrm{CI}=0.36,2.24) ;$ MCM8.3 showed fold change of $1.12(p=0.5961, \mathrm{CI}=0.71,1.77)$, and MCM9 showed fold change of $0.61(p=0.0974, \mathrm{CI}=0.33,1.11)$.

\section{b Delta CT for MCM8.3}

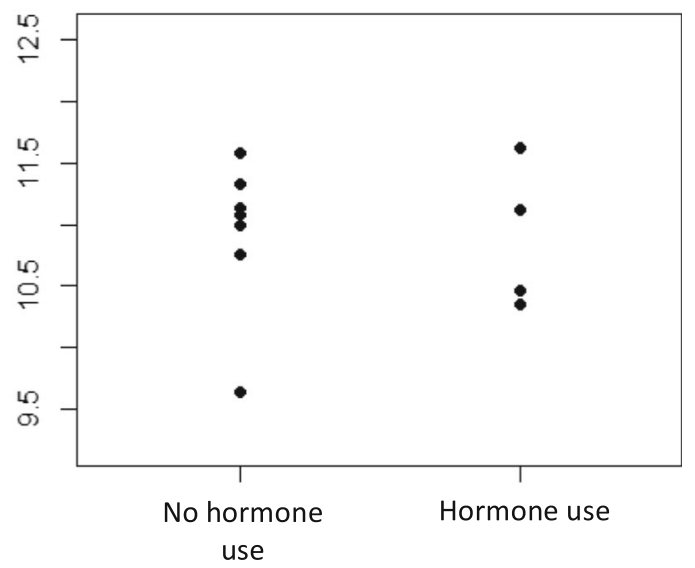

Fig. 3 Delta Ct for primers MCM 8.1 (a), MCM 8.3 (b), and MCM9.1 (c) in 7 subjects without hormonal contraception, vs 4 subjects that used hormonal contraception 
Delta CT for MCM8.1 by POI

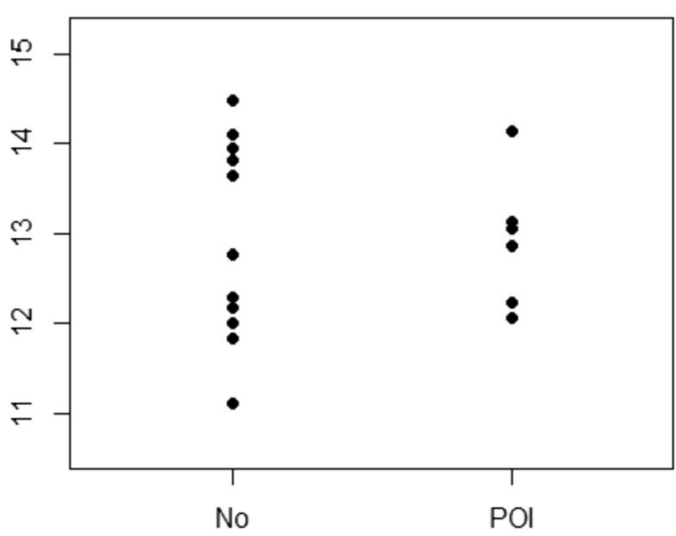

Delta CT for MCM9.1 by POI

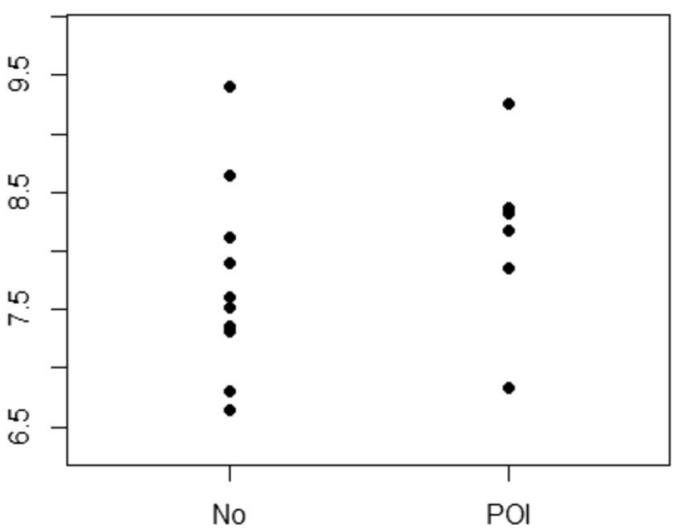

Delta CT for MCM8.3 by POI

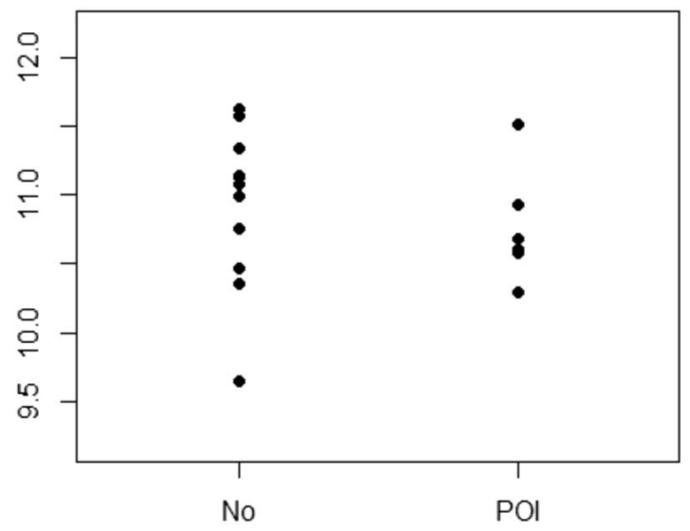

Fig. 4 Delta Ct for follicular phase for primers MCM 8.1 (a), MCM 8.3 (b), and MCM9.1 (c) in 11 controls in the follicular phase vs 6 subjects with POI

No statistically significant differences were noted when comparing amenorrheic POI subjects to normal controls in the ovulatory and luteal phases $(n=5)$; however, ovulatory values for both $M C M 8.3$ and 9 trend toward significance. MCM8.1 showed $\mathrm{FC}=1.16(p=0.734, \mathrm{CI}=0.44-3.03)$ at ovulation and $\mathrm{FC}=1.3(p=0.460, \mathrm{CI}=0.6-2.81)$ in the luteal phase. $M C M 8.3$ showed $\mathrm{FC}=1.53(p=0.104, \mathrm{CI}=0.9-2.62)$ at ovulation and $\mathrm{FC}=1.45(p=0.115, \mathrm{CI}=0.9-2.35)$ in the luteal phase. $M C M 9$ showed $\mathrm{FC}=0.56(p=0.059, \mathrm{CI}=0.31$, $1.03)$ at ovulation, and $\mathrm{FC}=0.79(p=0.466, \mathrm{CI}=0.38,1.61)$ in the luteal phase.

\section{Discussion}

The goal of our pilot study was to characterize gene expression of human MCM8 and MCM9 during the menstrual cycle in normo-ovulatory women. Additionally, we sought to compare MCM8 and 9 gene expression in females with unexplained primary ovarian insufficiency with normo-ovulatory, fertile controls in the follicular phase. Our findings demonstrated an increased $M C M 8$ expression in the follicular phase of the menstrual cycle; however, no change in $M C M 9$ expression across the follicular, ovulatory, and luteal phases. No significant differences were noted in MCM8 or MCM9 expression in POI subjects compared with normal controls.

MCM 8 and MCM9 form a complex that is needed for double-strand break (DSB) repair in prophase I. As oocytes in the human ovary are arrested in prophase I, and resume meiosis just prior to ovulation, we hypothesized that we would see increased gene expressions in the follicular or ovulatory phase of the menstrual cycle. A significant increase in MCM8 expression was noted in the follicular phase, compared with the ovulatory and luteal phases. However, there was no consistent variation of MCM9 gene expression seen in the menstrual cycle. Examining this more closely, homologous recombination occurs in the pachytene phase of prophase I, followed by the first meiotic arrest in the diplotene phase. The findings of our study may be due to the fact that the MCM8/9 complex in the ovary is needed to resolve DSB prior to the first meiotic arrest [5]. Supporting this concept, $M c m 8$ knockout mice show arrested primary follicles, whereas $\mathrm{Mcm} 9$ knockout 
mice have ovaries devoid of oocytes [5]. MCM8 may play a greater role in early follicular recruitment and development while $M C M 9$ is involved at an earlier/upstream step of germ cell proliferation, such that we did not see greater MCM9 expression in the follicular phase.

Alternatively, if menstrual cycle changes in $M C M$ expression are more notable in ovarian tissue, our results may be affected by the fact that we analyzed peripheral blood. We believed detection of MCM8 expression related to ovarian function using peripheral blood samples would be a validated and feasible approach based on a previous study of a consanguineous Arabic family with the MCM8 c.1954-1 G > A splice mutation [9]. This study demonstrated a threefold lower peripheral blood MCM8 expression (detected using a similar qRT-PCR technique) in two homozygous family members with primary amenorrhea due to gonadal failure, when compared to three unrelated "wild-type" control subjects. Three family members heterozygous for this same mutation showed an "intermediate" decrease in $M C M 8$ expression compared to controls. Nevertheless, it is possible this sampling approach may fail to detect subtle RNA expression changes outside the setting of a variant. Additionally, the prior study validated detecting $M C M 8$ expression changes in peripheral blood in subjects with $M C M 8$ mutation, but not necessarily MCM9 expression. This may explain why we were able to detect a difference in MCM8 expression, but not MCM9 expression.

In the future, it would be useful if comparison could be made between gene expression in the peripheral blood vs that in the ovary or granulosa cells. Although it would rarely be feasible to obtain multiple human ovarian samples in the course of a single menstrual cycle, comparison could be made between blood and granulosa cell expression at a single time point (i.e., using follicular fluid from oocyte aspiration in IVF patients).

In addition to their role in ovarian aging, there is evidence of the importance of $M C M 8$ and 9 in multiple organ systems, including the nervous system, immune system, skin, bone marrow, and endocrine system [15]. Mouse models have confirmed that MCM8 and 9 play a role in the normal growth and stability of mitotically active somatic cells [5]. Clinical phenotypes seen in homozygous MCM8 and MCM9 mutations include individuals affected with growth retardation and hypothyroidism, as well as infantile uteri and primary ovarian insufficiency. Perhaps most importantly, individuals affected with variants in these DSB repair genes have chromosomal instability in somatic cells, namely in fibroblasts and Tlymphocytes exposed to DNA cross-linking agents [14]. In mouse models, this chromosomal instability is associated with tumor development [5]. More recently, overexpression of MCM proteins has been noted in multiple cancers, including breast and uterine cancers [16].

A strength of our pilot study is that no prior studies at this time have investigated $M C M 8$ and 9 in the menstrual cycle. Characterizing $M C M$ expression changes in female circulation is vital as MCM proteins are currently being investigated as biomarkers for various forms of malignancy, due to the findings described above. As we have shown that at least MCM8 expression is altered during the menstrual cycle, it will be important for future researchers to take note of cycle phase when testing female subjects.

Small sample size was a limitation of our study. As the $p$ value and confidence interval were beginning to approach statistical significance, it is possible we would see a difference in MCM9 expression between our POI and control subjects with a larger study population. Further study is required to determine if such a difference could exist at a larger sample size. The effect of hormonal contraception on gene expression should also be investigated with a larger sample size, as it was not our initial objective to study this. Of our four study subjects on hormonal contraception, three used a progestin IUD and one used oral contraceptives. In theory, a progestin IUD has a primarily localized effect on the uterus and these subjects were still having monthly menses; therefore, it makes sense that their gene expression results were similar to the noncontraceptive users. However, oral contraceptives have a different mechanism of action, suppressing the hypothalamicpituitary-ovarian axis through negative feedback and preventing ovulation. This could potentially impact the expression of DNA repair genes.

\section{Conclusion}

In conclusion, our pilot study demonstrated greater $M C M 8$ gene expression in the follicular phase of the menstrual cycle, when compared to the ovulatory and luteal phases which may reflect a role in early follicular recruitment. This cyclical change was not seen with MCM9. No differences were noted in MCM8 or 9 gene expression in POI subjects vs normoovulatory controls; however, further investigation with a larger study population is recommended.

\section{Compliance with ethical standards}

Ethical approval All procedures performed in studies involving human participants were in accordance with the ethical standards of the institutional and/or national research committee and with the 1964 Helsinki declaration and its later amendments or comparable ethical standards.

Informed consent Informed consent was obtained from all individual participants included in the study.

\section{References}

1. Strauss J, Barbieri R, editors. Yen and Jaffe's reproductive endocrinology: physiology, pathophysiology and clinical management. Seventh ed. Philadephia PA: Elsevier Saunders; 2014. 
2. Perry JR, Corre T, Esko T, Chasman DI, Fischer K, Franceschini N, et al. A genome-wide association study of early menopause and the combined impact of identified variants. Hum Mol Genet. 2013;22(7):1465-72.

3. Stolk L, Perry JR, Chasman DI, He C, Mangino M, Sulem P, et al. Meta-analyses identify 13 loci associated with age at menopause and highlight DNA repair and immune pathways. Nat Genet. 2011;44(3):260-8.

4. Qin Y, Jiao X, Simpson JL, Chen ZJ. Genetics of primary ovarian insufficiency: new developments and opportunities. Hum Reprod Update. 2015;21(6):787-808.

5. Lutzmann M, Grey C, Traver S, Ganier O, Maya-Mendoza A, Ranisavljevic N, et al. MCM8- and MCM9- deficient mice reveal gametogenesis defects and genome instability due to impaired homologous recombination. Mol Cell. 2012;47(4):523-34.

6. Schuh-Huerta SM, Johnson NA, Rosen MP, Sternfeld B, Cedars MI, Reijo Pera RA. Genetic markers of ovarian follicle number and menopause in women of multiple ethnicities. Hum Genet. 2012;131:1709-24.

7. Nelson LM. Clinical practice. Primary ovarian insufficiency. N Engl J Med. 2009;360(6):606-14.

8. Desai S, Wood-Trageser M, Matic J, Chipkin J, Jiang H, Bachelot A, et al. MCM8 and MCM9 nucleotide variants in women with primary ovarian insufficiency. J Clin Endocrinol Metab. 2017;102:576-82.

9. Tenenbaum-Rakover Y, Weinberg-Shukron A, Renbaum P, Lobel $\mathrm{O}$, Eideh H, Gulsuner S, et al. Minichromosome maintenance complex component 8 (MCM8) gene mutations result in primary gonadal failure. J Med Genet. 2015;52:391-9.

10. AlAsiri S, Basit S, Woods-Trageser MA, Yatsenko SA, Jeffries EP, Surti U, et al. Exome sequencing reveals MCM8 mutation underlies ovarian failure and chromosomal instability. J Clin Invest. 2015;125:258-62.

11. Dou X, Guo T, Li G, Zhou L, Qin Y, Chen Z. Minichromosome maintenance complex component 8 mutations cause primary ovarian insufficiency. Fertil Steril. 2016;106(6):1485-9.

12. Bouali N, Francou B, Bouligand J, Lakhal B, Malek I, Kammoun $\mathrm{M}$, et al. New MCM8 mutation associated with premature ovarian insufficiency and chromosomal instability in a highly consanguineous Tunisian family. Fertil Steril. 2017;108(4):694-702.

13. Fauchereau F, Shalev S, Chervinsky E, Beck-Fruchter R, Legois B, Fellous M, et al. A non-sense MCM9 mutation in a familial case of primary ovarian insufficiency. Clin Genet. 2016;89:603-7.

14. Woods-Tragesar M, Gurbuz F, Yatsenko SA, Jeffries EP, Kotan LD, Surti U, et al. MCM9 mutations are associated with ovarian failure, short stature and chromosomal instability. Am J Hum Genet. 2014;95(6):754-62.

15. Yatsenko SA, Rajkovic A. Reproductive aging and MCM8/9. Oncotarget. 2015;6(18):15750-1.

16. He DM, Ren BG, Liu S, Tan LZ, Cieply K, Tseng G, et al. Oncogenic activity of amplified miniature chromosome maintenance 8 in human malignancies. Oncogene. 2017;36(25):3629-39. 